\title{
FVDZ-Bundesvorstandsmitglied van Rijt ist neuer ProDente-Vorsitzender
}

Hubertus van Rijt, Mitglied im Bundesvorstand des Freien Verbandes Deutscher Zahnärzte (FVDZ), ist zum neuen Vorstandsvorsitzenden der Initiative ProDente e.V. gewählt worden. Van Rijt folgt im Amt auf den bisherigen Vorstand Joachim Hoffmann und war vom FVDZ als Vorstandsmitglied nominiert worden. „Joachim Hoffmann hat die Initiative in den vergangenen Jahren sehr erfolgreich geführt", dankte ProDente-Geschäftsführer Dirk Kropp dem scheidenden Vorsitzenden. Das FVDZ-Bundesvorstandsmitglied van Rijt

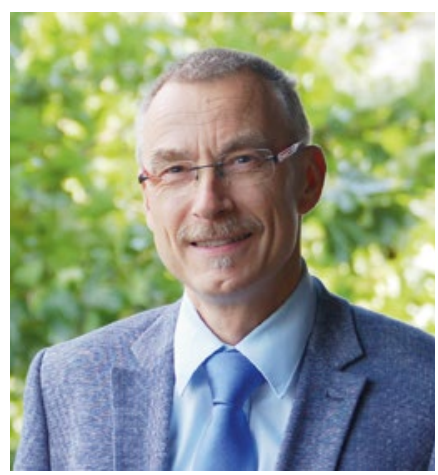

wurde einstimmig zum Vorstand des Vereins gewählt. Der gebürtige Niederländer ist seit fast 20 Jahren in eigener Praxis in Bielefeld niedergelassen. Die Initiative Prodente wird seit 1998 unter anderem vom FVDZ finanziert und vermittelt Fachwissen aus den Bereichen Zahnmedizin und Zahntechnik. Vor allem Aufklärungsarbeit von der Prophylaxe bis zur Prothetik steht dabei im Mittelpunkt. ProDente bietet dazu ein umfangreiches Serviceangebot für Zahnarztpraxen, um die Kommunikation mit den Patienten zu erleichtern. red/sas

\section{Carl-Erich-Alken-Medaille}

\section{Neue Auszeichnung für Dr. Gisela Tascher}

Für ihre unermüdlichen Arbeiten über die Rolle der Ärzteschaft im Nationalsozialismus hat die Zahnärztin Dr. Gisela Tascher einen weiteren Preis erhalten: Die Ärztekammer Saarland zeichnete sie mit der Carl-Erich-Alken-Medaille aus. Tascher, die auch seit Langem im Freien Verband Deutscher Zahnärzte aktiv ist, bekam für ihre historischen Aufarbeitungen bereits die Ehrennadel der Bundeszahnärztekammer sowie den HerbertLewin-Forschungspreis.
Außerdem ist sie als Vertreterin des Arbeitskreises Geschichte der Zahnmedizin in der Deutschen Gesellschaft für Zahn-, Mund- und Kieferheilkunde in das Aufarbeitungsprojekt zur Rolle des Zahnärztlichen Berufsstands im „Dritten Reich“ eingebunden, das von der Bundeszahnärztekammer, der Kassenzahnärztlichen Bundesvereinigung und der Deutschen Gesellschaft für Zahn-, Mund- und Kieferheilkunde finanziert wird.

\section{Mit wissen kompakt das Jahr informativ und zukunftsweisend beenden}

Durch den demographischen Wandel wird die zahnmedizinische Praxis zukünftig vermehrt mit zwei großen Gruppen an älteren Menschen zu tun haben: den rüstigen und den pflegebedürftigen Patienten. Der Umgang mit multimorbiden Patienten, die Einschätzung altersbedingter Veränderungen in der Mundhöhle und die Umsetzung altersgerechter Therapiekonzepte sind nur einige der Herausforderungen, denen sich die zahnmedizinische Versorgung vermehrt stellen muss. Die November-Ausgabe von wissen kompakt widmet sich daher dem wichtigen und zukunftsweisenden Thema „der alternde Patient“ in kompakter Form aufbereitet.

„Leichte kognitive Beeinträchtigung und beginnende Demenzen: eine klinische Übersicht" von C. Frankenberg, M.A., C. Degen, M. Sc., Prof. Dr. J. Schröder

"Alterszahnheilkunde - ein erfolgreiches Praxiskonzept" von Dr. E. Ludwig

"Altwerden und seine Bedeutung für Mundhöhle und Zähne" von Prof. Dr. W. Götz
„Mundgesundheit und Lebensqualität beim alternden Patienten" von Dr. A. Zenthöfer, Prof. Dr. A. J. Hassel, Prof. Dr. P. Rammelsberg

Die Teilnahme an den Continuing-Medical-Education(CME)Fortbildungen erfolgt online auf www.springerZahnmedizin.de und ist für die Mitglieder des Freien Verbandes Deutscher Zahnärzte (FVDZ) sowie für Abonnenten von wissen kompakt kostenlos. Beides trifft nicht auf Sie zu? Die entsprechenden Anträge finden Sie in dieser Ausgabe ab Seite 51.

wissen kompakt - gebündelte zertifizierte zahnärztliche Fortbildung

Im

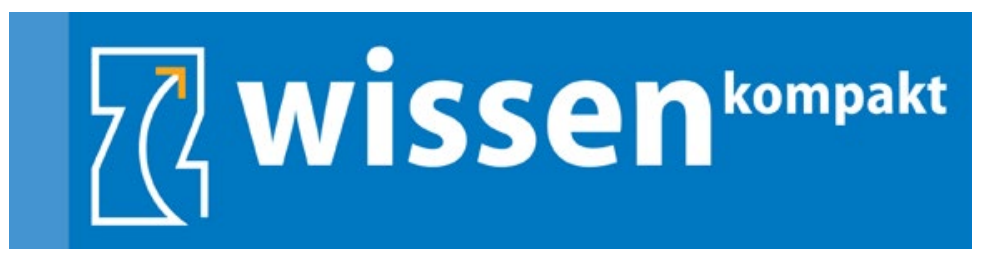

